\title{
On Characterizations of Randomly Censored Generalized Exponential Distribution
}

\author{
G. G. HAMEDANI
}

\begin{abstract}
The problem of characterizing a distribution is an important problem which has recently attracted the attention of many researchers. Thus, various characterizations have been established in many different directions. An investigator will be vitally interested to know if their model fits the requirements of a particular distribution. To this end, one will depend on the characterizations of this distribution which provide conditions under which the underlying distribution is indeed that particular distribution. In this work, several characterizations of Randomly Censored Generalized Exponential (RCGE) distribution are presented. These characterizations are based on: $(i)$ conditional expectation of certain functions of the random variable, $(i i)$ a single function of the random variable, (iii) the hazard function of the random variable.
\end{abstract}

\section{INTRODUCTION}

Characterizations of distributions are important to many researchers in the applied fields. An investigator will be vitally interested to know if their model fits the requirements of a particular distribution. To this end, one will depend on the characterizations of this distribution which provide conditions under which the underlying distribution is indeed that particular distribution. Various characterizations of distributions have been established in many different directions. In this work, several characterizations of Randomly Censored Generalized Exponential (RCGE) distribution are presented. These characterizations are based on: (i) conditional expectation of certain functions of the random variable, (ii) a single function of the random variable, ( $i i i)$ the hazard function of the random variable. For a detailed treatment of the RCGE distribution, we refer the interested reader to Danish and Aslam [1].

In [1] , the authors take the random variables $X$ and $T$ to be two independent random variables with $c d f^{\prime} s$ (cumulative distribution functions) $F_{X}(x)$ and $G_{T}(t)$ 
such that

$$
1-G_{T}(y)=\left\{1-F_{X}(y)\right\}^{\beta}
$$

where $\beta>0$ is a constant. Then, they choose the independent random variables $Y$ and $D$ as follows:

$$
1-F_{Y}(t)=\left(1-F_{X}(t)\right)\left(1-G_{T}(t)\right)
$$

and

$$
D=P(X \leq T)
$$

where $F_{Y}(t)$ is $c d f$ of $Y$.

Assuming that $X$ and $T$ are positive continuous random variables, the joint $p d f$ (probability density function) of $Y$ and $D$ is given by (see [1], page 49, (3))

$$
f_{Y, D}(y, d)=f_{X}(y)\left\{1-F_{X}(y)\right\}^{\beta}, \quad y>0, d=0,1 .
$$

Choosing $\quad F_{X}(x)=F_{X}(x ; \theta, \lambda)=\left(1-e^{-\lambda x}\right)^{\theta}$, we have from (1.1)

$f_{Y, D}(y, d ; \theta, \lambda, \beta)=\theta \lambda\left(1-e^{-\lambda y}\right)^{\theta-1} e^{-\lambda y}\left\{1-\left(1-e^{-\lambda y}\right)^{\theta}\right\}^{\beta} \beta^{1-d}, y>0, d=0,1$

The $p d f$ and $c d f$ of $Y$ are

$f_{Y}(y ; \theta, \lambda, \beta)=(\beta+1) \theta \lambda\left(1-e^{-\lambda y}\right)^{\theta-1} e^{-\lambda y}\left\{1-\left(1-e^{-\lambda y}\right)^{\theta}\right\}^{\beta}, y>0$,

and

$$
F_{Y}(y ; \theta, \lambda, \beta)=1-\left\{1-\left(1-e^{-\lambda y}\right)^{\theta}\right\}^{\beta+1}, \quad y \geq 0
$$

A random variable with $p d f$ and $c d f$ given by (1.3) and (1.4), respectively, is called RCGE random variable. 


\section{CHARACTERIZATIONS}

In this section, several characterizations of RCGE distribution are presented. These characterizations are based on: $(i)$ conditional expectation of certain functions of the random variable, (ii) a single function of the random variable, (iii) the hazard function of the random variable.

\subsection{Characterization based on the conditional expectation}

In this subsection we present characterizations of $p d f$ given by (1.3) in terms of conditional expectation of certain function of the RCGE random variable. We like to mention here the works of Glänzel and Hamedani [3] and Hamedani [4 - 7] as well as references therein, in this direction. Our characterization results presented in this subsection will employ a special version of an interesting result due to Glänzel [2] (Theorem 2.1.1 below).

THEOREM 2.1.1. Let $(\Omega, \mathcal{F}, \mathbf{P})$ be a given probability space and let $H=[a, b]$ be an interval for some $a<b$ ( $a=-\infty, b=\infty$ might as well be allowed). Let $Y: \Omega \rightarrow H$ be a continuous random variable with the distribution function $F$ and let $g$ be a real function defined on $H$ such that

$$
\mathbf{E}[g(Y) \mid Y \geq y]=\eta(y), \quad y \in H,
$$

is defined with some real function $\eta$. Assume that $g \in C^{1}(H), \eta \in C^{2}(H)$ and $F$ is twice continuously differentiable and strictly monotone function on the set $H$. Finally, assume that the equation $\eta=g$ has no real solution in the interior of $H$. Then $F$ is uniquely determined by the functions $g$ and $\eta$, particularly

$$
F(y)=\int_{a}^{y} C\left|\frac{\eta^{\prime}(u)}{\eta(u)-g(u)}\right| \exp (-s(u)) d u,
$$

where the function $s$ is a solution of the differential equation $s^{\prime}=\frac{\eta^{\prime}}{\eta-g}$ and $C$ is a constant, chosen to make $\int_{H} d F=1$. 
REMARK 2.1.2. In Theorem 2.1.1, the interval $H$ need not be closed.

PROPOSITION 2.1.3. Let $Y: \Omega \rightarrow(0, \infty)$ be a continuous random variable and let $g(y)=\left\{1-\left(1-e^{-\lambda y}\right)^{\theta}\right\}$ for $x \in(0, \infty)$. The $p d f$ of $Y$ is (1.3) if and only if the function $\eta$ defined in Theorem 2.1.1 has the form

$$
\eta(y)=\frac{\beta+1}{\beta+2}\left\{1-\left(1-e^{-\lambda y}\right)^{\theta}\right\}, \quad y \in(0, \infty) .
$$

PROOF. Let $Y$ have $p d f(1.3)$, then

$$
\mathbf{E}[g(Y) \mid Y \geq y]=\frac{\beta+1}{\beta+2}\left\{1-\left(1-e^{-\lambda y}\right)^{\theta}\right\}, \quad y \in(0, \infty),
$$

and

$$
\eta(y)-g(y)=-\frac{1}{\beta+2}\left\{1-\left(1-e^{-\lambda y}\right)^{\theta}\right\}<0, \quad y \in(0, \infty) .
$$

Conversely, if $\eta$ is given as above, then

$$
s^{\prime}(y)=\frac{\eta^{\prime}(y)}{\eta(y)-g(y)}=\frac{(\beta+1) \theta \lambda e^{-\lambda y}\left(1-e^{-\lambda y}\right)^{\theta-1}}{\left\{1-\left(1-e^{-\lambda y}\right)^{\theta}\right\}}, \quad y \in(0, \infty)
$$

and hence

$$
s(y)=-(\beta+1) \ln \left\{1-\left(1-e^{-\lambda y}\right)^{\theta}\right\}, \quad y \in(0, \infty) .
$$

Now, in view of Theorem 2.1.1, $Y$ has $c d f$ (1.4) and $p d f$ (1.3).

COROLLARY 2.1.4. Let $Y: \Omega \rightarrow(0, \infty)$ be a continuous random variable. The $p d f$ of $Y$ is (1.3) if and only if there exist functions $g$ and $\eta$ defined in Theorem 2.1.1 satisfying the differential equation

$$
\frac{\eta^{\prime}(y)}{\eta(y)-g(y)}=\frac{(\beta+1) \theta \lambda e^{-\lambda y}\left(1-e^{-\lambda y}\right)^{\theta-1}}{\left\{1-\left(1-e^{-\lambda y}\right)^{\theta}\right\}}, \quad y \in(0, \infty) .
$$

REMARKS 2.1.5. (b) The general solution of the differential equation in Corollary 2.1.4 is

$$
\eta(y)=\left\{1-\left(1-e^{-\lambda y}\right)^{\theta}\right\}^{-(\beta+1)}\left[-\int g(y)(\beta+1) \theta \lambda e^{-\lambda y}\left(1-e^{-\lambda y}\right)^{\theta-1}\left\{1-\left(1-e^{-\lambda y}\right)^{\theta}\right\}^{\beta+1} d y+D\right]
$$


for $x \in(0, \infty)$, where $D$ is a constant. One set of appropriate functions is given in Proposition 2.1.3 with $D=0$.

(c) Clearly there are other pairs of functions $(g, \eta)$ satisfying the conditions of Theorem 2.1.1. We presented one such pair in Proposition 2.1.3.

2.2. Characterization based on conditional expectation of single function of the random variable

In this subsection we employ a single function $\psi$ of $Y$ and characterize the distribution of $Y$ in terms of the conditional expectation of $\psi(Y)$. The following propositions have already appeared in our previous work, so we will just state them here for the sake of completeness.

PROPOSITION 2.2.1. Let $Y: \Omega \rightarrow(a, b)$ be a continuous random variable with $c d f F$. Let $\psi(y)$ be a differentiable function on $(a, b)$ with $\lim _{y \rightarrow a} \psi(y)=1$. Then for $\delta \neq 1$,

$$
E[\psi(Y) \mid Y>y]=\delta \psi(y), \quad y \in(a, b)
$$

if and only if

$$
\psi(y)=(1-F(y))^{\frac{1}{\delta}-1}, \quad y \in(a, b)
$$

PROPOSITION 2.2.2. Let $Y: \Omega \rightarrow(a, b)$ be a continuous random variable with $c d f F$. Let $\psi_{1}(y)$ be a differentiable function on $(a, b)$ with $\lim _{y \rightarrow b} \psi_{1}(y)=1$. Then for $\delta_{1} \neq 1$,

$$
E\left[\psi_{1}(Y) \mid Y<y\right]=\delta_{1} \psi_{1}(y), \quad y \in(a, b)
$$

if and only if

$$
\psi_{1}(y)=(F(y))^{\frac{1}{\delta_{1}}-1}, \quad y \in(a, b)
$$


REMARKS 2.2.3. (d) For $\psi(y)=\left\{1-\left(1-e^{-\lambda y}\right)^{\theta}\right\}^{\frac{(\beta+1)(1-\delta)}{\delta}}, y \in$ $(0, \infty)$, Proposition 2.2.1 will give a $c d f F(y)$ given by (1.4). (e) For $\psi_{1}(y)=\left\{1-\left\{1-\left(1-e^{-\lambda y}\right)^{\theta}\right\}^{\beta+1}\right\}^{\frac{1-\delta_{1}}{\delta_{1}}}, x \in(0, \infty)$, Proposition 2.2.2 will give a cdf $F(y)$ given by (1.4).

\subsection{Characterization based on hazard function}

For the sake of completeness, we state the following definition.

DEFINITION 2.3.1. Let $F$ be an absolutely continuous distribution with the corresponding $p d f f$. The hazard function corresponding to $F$ is denoted by $h_{F}$ and is defined by

$$
h_{F}(y)=\frac{f(y)}{1-F(y)}, \quad y \in \operatorname{Supp} F,
$$

where $\operatorname{Supp} F$ is the support of $F$.

It is obvious that the hazard function of twice differentiable function satisfies the first order differential equation

$$
\frac{h_{F}^{\prime}(y)}{h_{F}(y)}-h_{F}(y)=q(y)
$$

where $q(y)$ is an appropriate integrable function. Although this differential equation has an obvious form since

$$
\frac{f^{\prime}(y)}{f(y)}=\frac{h_{F}^{\prime}(y)}{h_{F}(y)}-h_{F}(y)
$$

for many univariate continuous distributions (2.3.2) seems to be the only differential equation in terms of the hazard function. The goal of the characterization based on hazard function is to establish a differential equation in terms of hazard function, which has as simple form as possible and is not of the trivial form (2.3.2) . For some general families of distributions this may not be possible. Here, we present 
a characterization of a RCGE distribution based on a nontrivial differential equation in terms of the hazard function.

PROPOSITION 2.3.2. Let $Y: \Omega \rightarrow(0, \infty)$ be a continuous random variable. The $p d f$ of $Y$ is (1.3) if and only if its hazard function $h_{F}(y)$ satisfies the differential equation

$h_{F}^{\prime}(y)=\frac{1}{\theta(\beta+1)} h_{F}^{2}(y)\left\{\left(1-e^{-\lambda y}\right)^{-\theta}\left(\theta-e^{\lambda y}\right)+e^{\lambda y}\right\}, \quad 0<y<\infty$.

PROOF. If $Y$ has $p d f$ (1.3), then clearly (2.3.3) holds. Now, if (2.3.3) holds, then

$$
\frac{h_{F}^{\prime}(y)}{h_{F}^{2}(y)}=\frac{1}{\theta(\beta+1)}\left\{\frac{\theta-e^{\lambda y\left[1-\left(1-e^{-\lambda y}\right)^{\theta}\right]}}{\left(1-e^{-\lambda y}\right)^{\theta}}\right\}
$$

from which we have

$$
\frac{d}{d y}\left(\frac{1}{h_{F}(y)}\right)=\frac{d}{d y}\left[\frac{1}{\theta \lambda(\beta+1)} e^{\lambda y}\left(1-e^{-\lambda y}\right)^{-\theta+1}\left[1-\left(1-e^{-\lambda y}\right)^{\theta}\right]\right],
$$

or

$$
h_{F}(y)=\theta \lambda(\beta+1) e^{-\lambda y}\left(1-e^{-\lambda y}\right)^{\theta-1}\left[1-\left(1-e^{-\lambda y}\right)^{\theta}\right]^{-1}
$$

Integrating both sides of (2.3.4) from 0 to $y$, we arrive at

$$
-\ln (1-F(y))=-(\beta+1) \ln \left(\left\{1-\left(1-e^{-\lambda y}\right)^{\theta}\right\}\right)
$$

From the last equality, we obtain

$$
1-F(y)=\left\{1-\left(1-e^{-\lambda y}\right)^{\theta}\right\}^{\beta+1}, \quad y \geq 0 .
$$




\section{REFERENCES}

[1] Danish, M.Y. and Aslam, M., Bayesian analysis of randomly censored generalized exponential distribution, Austrian J. Statistics, 42 (2013), 47 - 62.

[2] Glänzel, W., A characterization theorem based on truncated moments and its application to some distribution families, Mathematical Statistics and Probability Theory (Bad Tatzmannsdorf, 1986), Vol. B, Reidel, Dordrecht, 1987, 75 - 84.

[3] Glänzel, W. and Hamedani, G.G., Characterizations of univariate continuous distributions, Studia. Sci. Math. Hungar., 37 (2001), $83-118$.

[4] Hamedani, G.G., Characterizations of Cauchy, normal and uniform distributions, Studia Sci. Math. Hungar. 28 (1993), $243-247$.

[5] Hamedani, G.G., Characterizations of univariate continuous distributions. II, Studia Sci. Math. Hungar. 39 (2002), $407-424$.

[6] Hamedani, G.G., Characterizations of univariate continuous distributions. III, Studia Sci. Math. Hungar. 43 (2006), $361-385$.

[7] Hamedani, G.G., Characterizations of continuous univariate distributions based on the truncated moments of functions of order statistics, Studia Sci. Math. Hungar. 47 (2010), $462-484$.

November, 2, 2013

\section{G.G. Hamedani}

Department of Mathematics, Statistics and Computer

Science

Marquette University

Milwaukee, Wisconsin 53201-1881 